\section{Physical Basis of Insect Repellency}

THE physical basis of insect repellency being unknown, the search for new repellents has had to be conducted on an essentially hit-or-miss basis. With the accumulation of evidence that the odours of substances may be correlated with the low-frequency, fundamental vibrations of their molecules ${ }^{1}$, it seemed worth while to examine the absorption of some typical insect repellents in the far infra-red part of the spectrum.

The following commercial insect-repellent substances were available : dimethyl phthalate, indalone, 2 -ethyl hexanediol-1,3, benzyl benzoate, $n$-propyl$\mathrm{N}, \mathrm{N}$-diethyl succinamate and butoxy propylene glycol. In order to increase the statistical weight of the results, additional substances were chosen on the basis of ready availability from the extensive list of repellent chemicals published by Morton, Travis and Linduska 2 .

These workers tested 6,241 chemicals as mosquito repellents. The tests were made by covering the forearms of test subjects from wrist to elbow with the chemical or with a treated cloth, and then exposing them in cages containing some thousands of unfed mosquitoes (A. aegypti). The substances were graded on the basis of the duration of protection they afforded, the most effective substances being placed in group 4 and the least effective in group 1 . This method of screening could only have been approximate, and individual substances may now and then have been wrongly classified (benzyl benzoate, for example, was placed in group 1 and has since found application as a repellent); yet a random selection of compounds from group 4 may be expected to consist mainly if not entirely of repellents, and a similar selection from group 1 will be mainly nonrepellent.

Fourteen substances showing grade 4 repellency were collected from available chemical stocks to give a test set of twenty repellents altogether. A control group of twenty non-repellent (group 1) substances was also assembled from available chemical stocks. Infra-red absorption curves were recorded in the region from 700 to $400 \mathrm{~cm}^{-1}$ using a Perkin-Elmer Model 21 infra-red spectrophotometer equipped with a potassium bromide prism and a linear wave-number cam. Liquids were examined as received. Solids were usually examined as mulls in 'Nujol' (which is transparent in this region) or, in a few cases, as compressed films with potassium bromide.

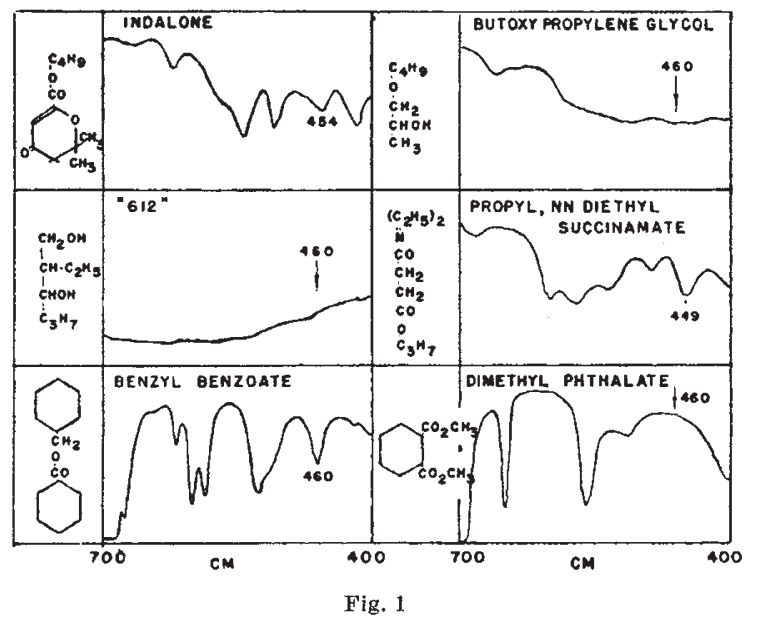

The absorption curves of the six commercial repellents are shown in Fig. 1. The results exhibit a striking correlation in the part of the spectrum near $460 \mathrm{~cm}^{-1}$. Of the whole group of twenty repellent substances, thirteen have peaks between 449 and $471 \mathrm{~cm}^{-1}$, three of the remainder show strong continuous absorption in this region, and three have nearby broad bands which overlap this region. Of the non-repellent substances, only one has a peak between 449 and $471 \mathrm{~cm}^{-1}$ and the rest with few exceptions show very little absorption in the neighbourhood of $460 \mathrm{~cm}^{-1}$.

The only marked exception in the repellent group is dimethyl phthalate, the repellent properties of which are well known; it has no absorption peak near $460 \mathrm{~cm} .^{-1}$. This substance is, however, on $\theta$ of the few members of the repellent group the structure of which is sufficiently symmetrical for certain vibrational modes to be inactive in the infra-red. It may be significant that the Raman spectrum of this substance $e^{3}$ has a line at $230 \mathrm{~cm}^{-1}$.

Thus the conclusion seems inescapable that the physical basis of mosquito repellency is a molecular vibrational mode capable of giving rise to infra-red absorption or Raman scattering near $460 \mathrm{~cm}^{-1}$ (or $21 \cdot 7 \mu$ ). Whether other species of insect are repelled by molecules exhibiting this or some other characteristic frequency is not yet known. The practical value of these results in screening new substances for mosquito repellency will be evident at once. Their bearing on the general problems of olfection is to lend support to the hypothesis that the lowfrequency vibrations of molecules provide the physical basis of their odours. A discussion of the mechanism whereby such vibrations may be able to trigger nerve discharges will be published elsewhere.

I thank the Van Ameringen Co., Firmenich, Inc., and Dr. Hubert Martin for the gift of samples.

Division of Chemistry,

\section{R. H. WRIGHT}

British Columbia Research Council,

Vancouver 8, Canada.

April 30.

${ }^{1}$ Wright, R. H., Nature, 173, 831 (1954); J. App. Chem., 4, 611 and 615 (1954).

2 Morton, F. A., Travis, B. V., and Linduska, J. P., U.s. Bur. Ent. and Plant Quarantine, September 1947 (E-733).

${ }^{3}$ Landolt-Bôrnstein, "Physikalisch-Chemisch Tabellen", Supp. 3, Pt. 2, 1158 (Springer Verlag, Berlin, 1935).

\section{Effect of Fungicides on Fungus Enzymes}

EvIDENCE has already been obtained to show that an important mechanism of the toxic action of fungicides is the inhibition of fungus enzymes. Copper and mercury are known to inhibit different systems, including those concerned with carbohydrate metabolism ${ }^{1}$. Rich and Horsfall ${ }^{2}$ have investigated the action of phenols and quinones upon polyphenol oxidase, while Owens ${ }^{3}$ has examined the effect of organo-sulphur compounds, phenols, quinones and metal compounds upon amylases, polyphenol oxidase and catalase. Attention has been given to the mode of action of different types of derivatives of thiocarbamic acid. Weed, McCallan and Miller ${ }^{4}$ found that ferbam (ferric dimethyldithiocarbamate) inhibited urease, whereas nabam (disodium ethylene bisdithiocarbamate) had little effect. Sisler and Cox ${ }^{5}$ attribute the toxicity of thiram (bis(dimethylthiocarbamoyl) disulphide) largely to the inhibition of the 